\title{
Mayor beneficio de los hipolipemiantes en diabéticos que en no diabéticos
}

Saha D, et al. N Engl J Med. 2006; 354:2452-2462.

\section{Objetivo}

Examinar el beneficio clínico del tratamiento con drogas hipolipemiantes en los pacientes diabéticos y no diabéticos, en prevención primaria y secundaria.

\section{Fuente de datos}

Los autores usaron las siguientes fuentes hasta abril de 2004: 1) Cochrane, 2) Medline, 3) Embase, 4) listas de referencias.

\section{Selección de estudios}

Seleccionaron todos los ensayos clínicos que habían comparado una droga hipolipemiante contra placebo en forma aleatorizada y doble ciego, con al menos 500 pacientes por rama, con análisis de los diabéticos en cada una de las ramas, con un seguimiento de por lo menos tres años y que evaluaran algún evento cardiovascular mayor del resultado primario combinado: muerte por enfermedad coronaria, infarto de miocardio (IM) no fatal o revascularización quirúrgica o endovascular. Los resultados secundarios fueron cada componentes por separado, accidente cerebrovascular (ACV) y cambios en las concentraciones de colesterol total, LDL, HDL y triglicéridos. Se incluyeron ensayos con pacientes con y sin enfermedad coronaria para evaluar la eficacia de la prevención primaria y secundaria. Dos autores evaluaron en forma independiente los estudios identificados en las bases de datos electrónicas seleccionando los que cumplieran con los criterios antedichos. Los desacuerdos se resolvieron por consenso. Se usó un paquete estadístico provisto por Cochrane y se testeó heterogeneidad. Se expresaron los resultados en forma de reducción del riesgo relativo* (RRR), analizándose por separado la prevención primaria y secundaria, la sub-población diabética y no diabética y el uso de estatinas y fibratos.

\section{Resultados}

Aplicando los criterios antedichos se incluyeron 12 ensayos: cuatro de prevención primaria, seis de secundaria y dos de ambas. Entre otros, estuvieron incluidos los estudios HPS, CARE y 4S. Se excluyó el estudio WOSCOPS debido a que no informó por sepa- rado los datos de la sub-población diabética. Los diabéticos tuvieron un mayor riesgo de eventos que los no diabéticos, tanto en las ramas placebo como en las de tratamiento, y tanto en prevención primaria como en secundaria. En cuanto al resultado primario, los hipolipemiantes parecen tener igual eficacia relativa en pacientes diabéticos y no diabéticos. Aunque se encontraron RRR similares las reducciones de riesgo absoluto (RRA) o beneficio atribuible son mayores y el número necesario para tratar (NNT) menores en diabéticos y en prevención secundaria.

Tabla 1: riesgo de evento cardiovascular mayor a un seguimiento promedio de 4,5 años.

\begin{tabular}{|c|c|c|c|c|c|c|c|c|c|c|}
\hline \multirow[b]{2}{*}{ Sub-población } & \multicolumn{5}{|c|}{ Prevención primaria } & \multicolumn{5}{|c|}{ Prevención secundaria } \\
\hline & Placebo & Droga & (ICRR & RRA & NNT & Placebo & Droga & \begin{tabular}{|l|l|} 
RRRR \\
(IC95\%
\end{tabular} & ARA & \\
\hline No diabếtica & 1243/15505 & & $21 \%$ & $1,07 \%$ & 93 & 3726/16801 & & $23 \%$ & 064 & \\
\hline Diabética & & $\begin{array}{c}\frac{6,31 \%}{433 / 5453} \\
\end{array}$ & $\begin{array}{l}(12-33) \\
21 \%\end{array}$ & $2,14 \%$ & 46 & 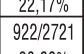 & $\begin{array}{l}\frac{11,111 \%}{743 / 2720} \\
\end{array}$ & $\begin{array}{c}(19-26) \\
19 \%\end{array}$ & $6,57 \%$ & 15 \\
\hline
\end{tabular}

En prevención secundaria se encontraron diferencias en la RRR de resultados secundarios entre diabéticos y no diabéticos. Para muerte por enfermedad coronaria las RRR fueron de 30\% (IC95\% 8-47) para diabéticos y $21 \%$ (5-35) para no diabéticos; para IM no fatal 39\% (4-62) para diabéticos y $29 \%$ (18-39) para no diabéticos; para procedimientos de revascularización 30\% (17-41) para diabéticos y $23 \%$ (18-27) para no diabéticos; para ACV 36\% (17-51) para diabéticos y $22 \%(13-30)$ para no diabéticos. Las magnitudes de cambio de los lípidos en sangre fueron similares en diabéticos y en no diabéticos con descensos de alrededor del $15 \%$ en el colesterol total y ascensos del $5 \%$ en el colesterol HDL. Los ensayos que usaron gemfibrozilo mostraron menor descenso del colesterol total.

\section{Conclusiones del metanálisis}

Los pacientes diabéticos se benefician más del tratamiento hipolipemiante que los pacientes no diabéticos, tanto en prevención primaria como secundaria.

Fuente de financiamiento: ninguna

\section{Comentario}

La diabetes es un factor de riesgo cardiovascular conocido que pone a los pacientes que la padecen en un riesgo de muerte por enfermedad cardiovascular cercano al de los pacientes no diabéticos que sufrieron un infarto de miocardio'. Dado que la mayoría de los pacientes diabéticos tiene un riesgo de desarrollar enfermedad coronaria a diez años mayor a $20 \%$ es que las guías de prácticas clínica sugieren activa intervención sobre los factores de riesgo de estos pacientes, incluyendo el uso de estatinas para lograr un descenso de los valores de LDL colesterol por debajo de $100 \mathrm{mg} \%$ en prevención primaria y a menos de $70 \mathrm{mg} \%$ en prevención secundaria-5. Este estudio se propone contestar la pregunta ¿los pacientes diabéticos reducen el riesgo cardiovascular en respuesta a la terapia hipolipemiante más, menos o igual que los no diabéticos? La respuesta, en términos de RRR, para el resultado primario, tanto para prevención primaria como secundaria es que lo reducen igual. Las objeciones ${ }^{6.9}$ pasan por: 1) la inclusión de ensayos que no usan estatinas, 2) el uso de la RRR para expresar los resultados cuando los riesgos atribuibles y su inversa el NNT difieren mucho, dada la desigualdad de lo riesgos basales en cada una de las poblaciones. ¿Es lícito decir que los diabéticos se benefician igual porque la RRR es similar? ¿Es correcto decir que los diabéticos se benefician más cuando ya, su riesgo basal es mayor?

\section{Conclusiones del comentador}

Los diabéticos se benefician más de la terapia hipolipemiante tanto en prevención primaria como secundaria, porque tienen más riesgo basal. Este estudio no agrega nada particular al conocimiento actual, sino que más bien confirma que cualquier paciente se beneficiará más o menos de la terapia hipolipemiante, de acuerdo a su riesgo cardiovascular.

Bernardo Martinez [ Servicio de Clínica Médica. Hospital Italiano de Buenos Aires. ] Recibido el 10 de julio de 2006 y aceptado el 14 de agosto de 2006. Martinez B. Mayor beneficio de los hipolipemiantes en diabéticos que en no diabéticos. Evid. actual. páct. ambul. 9(5);137. Sept-Oct. 2006. Comentado de: Costa Joao, Borges Margarita, David Claudio, Vaz Carneiro Antonio. Efficacy of lipid lowering drug treatment for diabetic and non-diabetic patients: metaanalysis of randomised controlled trials. BMJ 2006;332:1115-24. PMID: 16585050.

\section{Referencias}

1. Haffner SM, Lehto S, Ronnemaa T, Pyorala K, Laakso M. Mortality from coronary heart disease in subjects with type 2 diabetes and in nondiabetic subjects with and without prior myocardial interaction. Neng J Med. 1998;339:229-234.

2. Third Report of the National Cholesterol Education Program (NCEP) Expert Panel on Detection, Evaluation, and Treatment of High Blood Cholesterol in Adults (Adult Treatment Panel III) final report. Circulation 2002 Dec 17;106(25):3143-421

3. Implications of recent clinical trials for the National Cholesterol Education Program Adult Treatment Panel III guidelines. Grundy SM; Cleeman JI; Merz CN; Brewer HB Jr; Clark LT; Hunninghake DB; Pasternak RC; Smith SC Jr; Stone NJ. Circulation 2004 Jul 13;110(2):227-39.

4. MRC/BHF Heart Protection Study of cholesterol lowering with simvastatin in 20,536 high-risk individuals: a randomised placebo-controlled trial. Lancet 2002 Jul 6:360(9326):7-22.

5. Diabetes and lipid lowering: where are we? Reckless JPD. BMJ 2006 May 13; 1103-4

6. Limitations of meta-analysis may cloud primary outcomes differences. Bakhai A; BMJ rapid responses to Efficacy of lipid lowering drug treatment for diabetic and non-diabetic patients: meta-analysis of randomised controlled trials. BMJ 2006;332:1115-1124; BMJ 20064 APRIL

7. Meta-analysis of lipid lowering tratment: abstract misleading?. van Driel M; BMJ rapid responses to Efficacy of lipid lowering drug treatment for diabetic and 